\title{
DESENVOLVIMENTO MENTAL E MOTOR AOS 24 MESES DE CRIANÇAS NASCIDAS A TERMO COM BAIXO PESO
}

\author{
Sophie Helena Eickmann ${ }^{1}$, Pedro Israel Cabral de Lira², Marilia de Carvalho Lima ${ }^{3}$
}

\begin{abstract}
RESUMO - Este estudo teve como objetivo comparar o desenvolvimento aos 24 meses de 152 crianças nascidas a termo, com baixo peso $(<2500 \mathrm{~g})$ e peso adequado $(3000$ a $3499 \mathrm{~g})$, pareadas numa proporção de 1:1 por sexo e idade. 0 desenvolvimento mental e motor foram avaliados pela escala de Bayley. Estudaram-se ainda algumas variáveis ambientais, como as condições sócio-econômicas e demográficas e a estimulação ambiental. As crianças do grupo de baixo peso ao nascer apresentaram, para os índices mental e motor, média significantemente mais baixa do que a do grupo de peso adequado $(p<0,001)$ com diferença de 9,1 e 10,2 pontos, respectivamente. A análise de regressão linear múltipla evidenciou que as condições sócio-econômicas e de estimulação ambiental explicaram $11 \%$ e $12 \%$ da variação do índice de desenvolvimento mental e $12 \%$ e $9 \%$ do desenvolvimento motor, respectivamente. Juntas elas explicaram $23 \%$ e $21 \%$ da variação desses índices. 0 baixo peso ao nascer influenciou apenas 3\% da variação do índice mental e $5 \%$ do motor.
\end{abstract}

PALAVRAS-CHAVE: baixo peso ao nascer, retardo de crescimento intra-uterino, desenvolvimento mental, desenvolvimeto motor, estimulação ambiental.

Mental and motor development at 24 months of full-term low birthweight infants

ABSTRACT - The objective of this study was to compare the development at 24 months of 152 full-term infants, born with low $(<2500 \mathrm{~g})$ and appropriate birth weight $(3000$ to $3499 \mathrm{~g})$, paired in a proportion of 1:1 by sex and age. Mental and motor development were assessed through the Bayley scale. A variety of sociodemographic and environmental stimulation conditions were also assessed. The infants born with low weight had on average significantly lower mental and motor indexes than those born with appropriate weight $(p<0.001)$, with a difference of 9.1 and 10.2 points, respectively. The multiple linear regression analysis showed that socioeconomic conditions and environmental stimulation explained $11 \%$ and $12 \%$ of the variation of mental index, and $12 \%$ and $9 \%$ of motor development, respectively. All together, they explained $23 \%$ and $21 \%$ of the variation of these indexes. Low birth weight influenced only $3 \%$ of the variation of mental index and $5 \%$ of motor index.

KEY WORDS: low birth weight, intra-uterine growth retardation, mental development, motor development, environmental stimulation.

A prevalência de baixo peso ao nascer (BPN) varia muito entre os diversos países, sendo estimada para o Brasil como um todo em $9 \%{ }^{1}$. Essa variação é maior nos países em desenvolvimento, onde o percentual de crianças com BPN, mais frequentemente às custas de recém-nascido (RN) a termo, é maior e secundário a retardo do crescimento intra-uterino $(\mathrm{RCIU})^{2}$. O BPN está associado com incremento da morbidade ${ }^{3}$ e da mortalidade infantil ${ }^{4,5}$.
Apesar de ainda não haver consenso na literatu$\mathrm{ra}$, a maioria dos autores aceita o BPN em RN a termo como importante fator determinante do desenvolvimento, principalmente cognitivo e comportamental $^{6-9}$. A maior polêmica entre os autores diz respeito ao modo de atuação do RCIU sobre o sistema nervoso central e aos outros fatores que interferem neste processo, como o nível sócio-econômico e a estimulação ambiental ${ }^{10-12}$.

Centro de Ciências da Saúde da Universidade Federal de Pernambuco, Recife PE, Brasil: ${ }^{1}$ Neurologista do Desenvolvimento, Mestre em Pediatria; ${ }^{2}$ Professor Adjunto do Departamento de Nutrição, Doutor em Medicina; ${ }^{3}$ Professora Adjunta do Departamento MaternoInfantil, Doutora em Medicina.

Recebido 26 Novembro 2001, recebido na forma final 27 Março 2002. Aceito 15 Abril 2002.

Dra. Sophie Eickmann - Mestrado em Pediatria - Hospital das Clínicas, Bloco "A" Térreo - Avenida Professor Moraes Rego s/n - 50670-420 Recife PE - Brasil. E-mail: mped@npd.ufpe.br 
O presente estudo teve como objetivo comparar o desenvolvimento mental e motor aos 24 meses de crianças nascidas a termo com baixo peso e peso adequado, além de identificar alguns de seus fatores determinantes.

\section{MÉTODOS}

Este estudo consistiu de um corte transversal utilizando uma sub-amostra de um coorte prospectivo, de crianças acompanhadas do nascimento até os 24 meses de vida, cuja descrição detalhada foi publicada anteriormente ${ }^{3}$.

A pesquisa foi realizada em cinco cidades do interior do Estado de Pernambuco, onde a principal atividade econômica é a plantação e o processamento da cana de açúcar, $81 \%$ das famílias tendo renda mensal de até dois salários mínimos (1 SM = US\$ 70). Mais de 90\% dos partos das cinco cidades no período estudado ocorreram em maternidades e a incidência de BPN foi de 9,5\%. Todas as crianças desta pesquisa foram recrutadas em centros hospitalares, entre janeiro de 1993 e janeiro de 1994. Foram incluídas apenas crianças cujas famílias moravam na zona urbana, que tinham renda de até três salários mínimos mensais e que consentiram em participar do estudo.

Nas primeiras 24 horas de vida, todos os RN eram examinados por dois pediatras, sendo avaliados quanto às medidas antropométricas (peso, comprimento e perímetro cefálico) pela padronização da Organização Mundial da Saúde $(\mathrm{OMS})^{13}$ e quanto ao índice de maturidade pelo método de Capurro et al. ${ }^{14}$, sendo incluídos apenas aqueles com idade gestacional $\geq 37$ semanas.

Foram também excluídos os RN frutos de gestação gemelar, aqueles com evidências no pós-parto imediato de infecções congênitas, anomalias cromossômicas, malformações maiores, distúrbios neurológicos e/ou que necessitaram de cuidados intensivos neonatais.

O grupo de baixo peso foi composto de 76 bebês com peso ao nascer entre $1500 \mathrm{~g}$ e $2499 \mathrm{~g}$. Para o grupo controle pareou-se individualmente o primeiro bebê do mesmo sexo nascido após o caso, que apresentou peso entre $3000 \mathrm{~g}$ e $3499 \mathrm{~g}$ e que nasceu em média até 10 dias após o RN caso, totalizando uma amostra de 152 RN.

Este estudo foi aprovado pelo Comitê de Ética em Pesquisa do Centro de Ciências da Saúde da Universidade Federal de Pernambuco.

Avaliação do desenvolvimento - As crianças foram avaliadas com a idade de 24 meses +15 dias, na presença da mãe ou responsável, em uma sala especialmente preparada para tal finalidade, no Hospital da Fundação Nacional de Saúde, em Palmares. A avaliação do desenvolvimento foi realizada pela primeira autora utilizando a Escala Bayley de Desenvolvimento Infantil - versão 1984, com material padronizado original e seguindo as indicações do manual ${ }^{15}$. A avaliadora era cega quanto à condição do peso ao nascer.

Estimulação ambiental - Através de dados de anamnese realizada pela mesma autora, pesquisaram-se os vários tipos de estímulos que a criança recebia em seu ambiente familiar, adaptados do questionário $\mathrm{HOME}^{23}$ e agrupados em módulos: motor fino, motor amplo e verbalização. Sobre o desenvolvimento motor fino investigaram-se atividades de manipular brinquedos (construir e empilhar brinquedos pequenos, fazer quebra-cabeça) e pintar e/ou rabiscar. Entre os estímulos do desenvolvimento motor amplo, questionou-se sobre jogos motores como correr, pular, dançar e balançar. A pesquisa sobre os estímulos verbais incluiu atividades como conversar, mostrar figuras e ler histórias. Os estímulos ambientais prestados pela mãe ou outro adulto foram considerados como presentes quando ocorressem pelo menos três vezes por semana, por aproximadamente 30 minutos. Perguntou-se ainda sobre a ocorrência de castigos físicos na semana anterior ao exame. Por problemas logísticos, 26 crianças não foram avaliadas quanto à estimulação ambiental.

Avaliação do estado nutricional - A avaliação antropométrica das crianças aos 24 meses foi realizada por duas técnicas da equipe, devidamente treinadas. Os dados antropométricos foram aferidos utilizando-se as técnicas preconizadas pela $\mathrm{OMS}^{13}$. Para a classificação do estado nutricional, utilizou-se o padrão do NCHS - National Center for Health Statistics ${ }^{16}$.

Fatores sócio-econômicos e demográficos - Foi aplicado à mãe na maternidade um questionário para determinar alguns indicadores, entre eles a renda per capita, a co-habitação dos pais, a idade e o nível de escolaridade maternos e as condições de moradia, representadas pela presença de sanitário e geladeira ${ }^{3}$.

Análises estatísticas - As análises bivariadas foram realizadas com o EPI INFO versão 6.04 e utilizaram-se o teste do qui-quadrado e o t de Student, aceitando-se como significante um $p \leq 0,05$. A análise de regressão linear múltipla (método stepwise), através do Statistical Package for Social Science (SPSS - 8.0), foi utilizada para avaliar o impacto das variáveis independentes sobre o desenvolvimento aos 24 meses, assim como para controlar o efeito de possíveis fatores de confusão. As variáveis selecionadas para ser introduzidas no modelo de regressão linear foram aquelas cujo nível de significância estatística nas análises entre as variáveis explanatórias e os índices de desenvolvimento mental e motor foi $\leq 0,20$.

\section{RESULTADOS}

Da amostra de 152 crianças 64 (42\%) eram do sexo masculino e 88 (58\%) do feminino. As medidas antropométricas das crianças com BPN eram aquém das do grupo controle, sendo o peso médio do primeiro grupo $2332 \mathrm{~g}(\mathrm{DP}=160)$ e no grupo de PAN $3255 \mathrm{~g}(\mathrm{DP}=141)$.

Aos 24 meses de idade, essas crianças persistiram com a média de peso, comprimento e períme- 
tro cefálico mais baixas do que as das crianças do grupo de PAN (Tabela 1).

\section{Variáveis sócio-econômicas e demográficas}

Apesar da tentativa de homogeneizar a amostra, encontramos diferenças significantes, em alguns indicadores sócio-econômicos. O nível de educação materna diferiu significantemente sendo menor no grupo de BPN. Apesar da renda familiar per capita não ter mostrado diferença significante entre os dois grupos, a presença de geladeira no domicílio, como indicador indireto do nível sócio-econômico, foi me- nos frequente nas famílias com crianças de BPN. O percentual de mães adolescentes neste mesmo grupo foi significantemente mais elevado que no grupo controle. Em $28 \%$ das famílias de crianças de BPN, o pai não residia na mesma casa e esse percentual (13\%) foi significantemente menor no grupo de PAN. Maior percentual absuluto de residências sem sanitário ou com sanitário sem descarga (55\%) foi observado entre as crianças de BPN quando comparado com as de PAN (39\%), apesar de não ter sido esta diferença estatisticamente significante (Tabela 2).

Tabela 1. Média e desvio padrão das medidas antropométricas aos 24 meses, segundo o peso ao nascer.

\begin{tabular}{lccccc}
\hline Variáveis & \multicolumn{2}{c}{ Baixo peso } & \multicolumn{2}{c}{ Peso adequado } & Teste de \\
& \multicolumn{2}{c}{$\mathrm{N}=75$} & \multicolumn{2}{c}{$\mathrm{N}=73$} & significância \\
& Média & (DP) & Média & (DP) & \\
\hline Peso $(\mathrm{kg})$ & 10,7 & $(1,5)$ & 11,9 & $(1,3)$ & $\mathrm{t}=27,43$ \\
& & & & & $\mathrm{p}<0,001$ \\
Comprimento $(\mathrm{cm})$ & 81,9 & $(4,0)$ & 84,5 & $(3,6)$ & $\mathrm{t}=16,40$ \\
& & & & & $\mathrm{p}<0,001$ \\
& 46,8 & $(1,4)$ & 48,1 & $(1,3)$ & $\mathrm{t}=36,50$ \\
& & & & & $\mathrm{p}<0,001$ \\
\hline
\end{tabular}

Tabela 2. Fatores sócio-econômicos e demográficos segundo o peso ao nascer.

\begin{tabular}{|c|c|c|c|c|c|}
\hline \multirow[t]{2}{*}{ Variáveis } & \multicolumn{2}{|c|}{ Baixo peso } & \multicolumn{2}{|c|}{ Peso adequado } & \multirow{2}{*}{$\begin{array}{c}\text { Teste de } \\
\text { significância }\end{array}$} \\
\hline & $N=76$ & $(\%)$ & $N=76$ & $(\%)$ & \\
\hline \multicolumn{6}{|l|}{ Renda per capita } \\
\hline \multicolumn{6}{|l|}{ ( salário mínimo ) } \\
\hline$\leq 0,25$ & 51 & $(67,1)$ & 40 & $(52,6)$ & $\chi^{2}=2,74$ \\
\hline$>0,25$ & 25 & $(32,9)$ & 36 & $(47,4)$ & $p=0,09$ \\
\hline \multicolumn{6}{|c|}{ Alfabetização materna } \\
\hline \multirow[t]{2}{*}{ Não } & 20 & $(26,3)$ & 12 & $(15,8)$ & $\chi^{2}=1,94$ \\
\hline & & & & & $p=0,16$ \\
\hline \multicolumn{6}{|c|}{ Escolaridade materna (anos) } \\
\hline $0-4$ & 43 & $(56,6)$ & 28 & $(36,8)$ & $\chi^{2}=5,18$ \\
\hline$\geq 5$ & 33 & $(43,4)$ & 48 & $(63,2)$ & $p=0,02$ \\
\hline \multicolumn{6}{|l|}{ Idade materna (anos) } \\
\hline $13-19$ & 37 & $(48,7)$ & 14 & $(18,4)$ & $\chi^{2}=14,28$ \\
\hline$\geq 20$ & 39 & $(51,5)$ & 62 & $(81,6)$ & $p<0,001$ \\
\hline \multicolumn{6}{|l|}{ Co-habitação } \\
\hline Sim & 55 & $(72,4)$ & 66 & $(86,8)$ & $\chi^{2}=4,05$ \\
\hline Não & 21 & $(27,6)$ & 10 & $(13,2)$ & $p=0,04$ \\
\hline \multicolumn{6}{|l|}{ Sanitário } \\
\hline Sem descarga ou & 42 & $(55,3)$ & 30 & $(39,5)$ & $\chi^{2}=3,19$ \\
\hline sem sanitário & & & & & $p=0,07$ \\
\hline \multicolumn{6}{|l|}{ Geladeira } \\
\hline \multirow[t]{2}{*}{ Não } & 48 & $(63,2)$ & 37 & $(48,7)$ & $\chi^{2}=2,67$ \\
\hline & & & & & $p=0,01$ \\
\hline
\end{tabular}


Tabela 3 . Estímulos ambientais e castigo físico segundo o peso ao nascer.

\begin{tabular}{|c|c|c|c|c|c|}
\hline \multirow[t]{2}{*}{ Variáveis } & \multicolumn{2}{|c|}{ Baixo peso } & \multicolumn{2}{|c|}{ Peso adequado } & \multirow{2}{*}{$\begin{array}{c}\text { Teste de } \\
\text { significância }\end{array}$} \\
\hline & $N=64$ & $(\%)$ & $N=62$ & $(\%)$ & \\
\hline \multicolumn{6}{|l|}{ Motor fino } \\
\hline Manipular brinquedos & 12 & $(18,8)$ & 18 & $(29,0)$ & $\begin{array}{c}\chi^{2}=1,31 \\
p=0,25\end{array}$ \\
\hline Pintar/rabiscar & 43 & $(67,2)$ & 52 & $(83,9)$ & $\begin{array}{l}\chi^{2}=3,87 \\
p=0,05\end{array}$ \\
\hline \multicolumn{6}{|l|}{ Motor amplo } \\
\hline $\begin{array}{l}\text { Correr/dançar/ } \\
\text { Balançar }\end{array}$ & 61 & $(95,3)$ & 61 & $(98,4)$ & $\begin{array}{l}\text { T. exato de } \\
\text { Fisher } \\
p=0,61\end{array}$ \\
\hline \multicolumn{6}{|l|}{ Verbal } \\
\hline Conversar & 49 & $(76,6)$ & 54 & $(87,1)$ & $\begin{array}{l}\chi^{2}=1,69 \\
p=0,19\end{array}$ \\
\hline Contar histórias/ ler livros & 36 & $(56,2)$ & 40 & $(64,5)$ & $\begin{array}{l}\chi^{2}=0,59 \\
p=0,44\end{array}$ \\
\hline Castigo físico & 47 & $(73,4)$ & 43 & $(69,4)$ & $\begin{array}{l}\chi^{2}=0,10 \\
p=0,76\end{array}$ \\
\hline
\end{tabular}

Tabela 4. Média e desvio padrão dos índices de desenvolvimento mental e motor pela Escala de Bayley aos 24 meses segundo o peso ao nascer.

\begin{tabular}{|c|c|c|c|c|c|}
\hline \multirow[t]{2}{*}{ Variáveis } & \multicolumn{2}{|c|}{$\begin{array}{c}\text { Baixo peso } \\
N=76\end{array}$} & \multicolumn{2}{|c|}{$\begin{array}{l}\text { Peso adequado } \\
\qquad N=76\end{array}$} & \multirow[t]{2}{*}{$\begin{array}{c}\text { Teste de } \\
\text { significância }\end{array}$} \\
\hline & Média & (DP) & Média & (DP) & \\
\hline $\begin{array}{l}\text { Desenvolvimento } \\
\text { mental (índice) }\end{array}$ & 89,8 & $(15,8)$ & 98,9 & $(14,8)$ & $\begin{array}{l}t=13,28 \\
p<0,001\end{array}$ \\
\hline $\begin{array}{l}\text { Desenvolvimento } \\
\text { motor (índice) }\end{array}$ & 95,1 & $(19,2)$ & 105,3 & $(13,6)$ & $\begin{array}{l}t=14,43 \\
p<0,001\end{array}$ \\
\hline
\end{tabular}

\section{Estimulação ambiental}

A Tabela 3 mostra que os estímulos ambientais foram menos frequentes no grupo de BPN; apenas a atividade de pintar e/ou rabiscar apresentou diferença estatisticamente significante entre os dois gru$\operatorname{pos}(p=0,05)$.

\section{Desenvolvimento neuropsicomotor}

O desenvolvimento mental e motor aos 24 meses de idade, avaliado pela escala de Bayley, mostrou que as crianças do grupo de BPN apresentaram um índice, tanto do desenvolvimento mental como motor, significantemente mais baixo que o das crianças do grupo de PAN ( $p<0,001)$. A diferença média dos índices mental e motor entre os dois grupos foi 9,1 e 10,2 pontos, respectivamente (Tabela 4).

\section{Análise multivariada}

O peso ao nascer esteve significantemente associado a um déficit de desenvolvimento aos 24 meses de idade. Apesar da renda familiar ter sido um dos critérios de inclusão objetivando selecionar amostra mais homogênea em relação às condições sócio-econômicas, alguns de seus indicadores diferiram entre as crianças de BPN e de PAN. Procurou-se, então, através de análise multivariada, estudar o efeito conjunto do peso ao nascer e dos fatores sócio-econômicos sobre os índices de desenvolvimento mental e motor. Este tipo de análise permite verificar o efeito de cada uma das variáveis independentes, ajustado pelo efeito das demais. As variáveis que participaram dos modelos 1 e 2 foram: escolaridade e alfabetização materna, estado nutricional aos 24 meses, presença de TV no domicílio e outros itens da estimulação ambiental (pintar/rabiscar e correr/dançar/balançar). 
Tabela 5. Regressão linear múltipla entre o peso ao nascer, condição sócio-econômica e a estimulação ambiental e os índices de desenvolvimento mental e motor (Escala de Bayley) aos 24 meses.

\begin{tabular}{|c|c|c|c|c|c|c|c|c|}
\hline $\begin{array}{l}\text { Variáveis } \\
\text { dependentes }\end{array}$ & $\begin{array}{c}\text { Variáveis } \\
\text { independentes }\end{array}$ & B & IC & de & $95 \%$ & $P$ & $\mathrm{R}^{2}$ & $\begin{array}{c}\text { Variação } \\
\text { de } R^{2}\end{array}$ \\
\hline \multicolumn{9}{|l|}{ Modelo 1} \\
\hline Índice de & Geladeira & $+7,8$ & 2,6 & a & 13,1 & 0,004 & 0,11 & 0,11 \\
\hline desenvolvimento mental & Ler ou contar histórias & $+7,9$ & 2,6 & a & 13,2 & 0,004 & 0,19 & 0,08 \\
\hline \multirow[t]{2}{*}{$N=125$} & Manipular brinquedos & $+6,7$ & 0,5 & a & 12,8 & 0,03 & 0,23 & 0,04 \\
\hline & Baixo peso & $-6,0$ & $-0,8$ & a & $-11,2$ & 0,01 & 0,26 & 0,03 \\
\hline \multicolumn{9}{|l|}{ Modelo 2} \\
\hline Índice de & Geladeira & $+9,0$ & 3,9 & a & 14,1 & 0,001 & 0,12 & 0,12 \\
\hline desenvolvimento mental & Ler ou contar histórias & $+9,0$ & 3,9 & a & 14,2 & 0,001 & 0,21 & 0,09 \\
\hline$N=125$ & Baixo peso & $-7,4$ & $-2,3$ & $\mathrm{a}$ & $-12,5$ & 0,005 & 0,26 & 0,05 \\
\hline
\end{tabular}

O Modelo 1 da Tabela 5, apresenta a análise de regressão linear múltipla entre o baixo peso ao nascer, condição sócio-econômica, estimulação ambiental e o índice de desenvolvimento mental. A condição sócio-econômica, representada pela posse de geladeira na residência ( $11 \%$ ) e a estimulação ambiental (brincar com a criança - 4\% e ler ou contar histórias - $8 \%$ ) juntas, explicaram $23 \%$ da variação do índice mental aos 24 meses, enquanto o baixo peso ao nascer explicou apenas 3\% da variação deste índice. Outros fatores, ainda não devidamente conhecidos ou mensurados neste estudo, seriam responsáveis pelo restante da variabilidade no desenvolvimento mental aos 24 meses.

O Modelo 2 da Tabela 5 mostra que o fator que melhor explicou a variação do desenvolvimento motor aos 24 meses de idade foi o nível sócio-econômico, sendo cerca de $12 \%$ da variação deste índice explicada pela posse de geladeira no domicílio. Logo em seguida, vem a estimulação ambiental (ler ou contar histórias), explicando $9 \%$ desta variação e por último o baixo peso ao nascer responsável por $5 \%$ da variação do índice motor.

Em resumo, o peso ao nascer mostrou ter impacto significante sobre o desenvolvimento mental e motor aos 24 meses; no entanto, a condição sócioeconômica e a estimulação ambiental tiveram impacto maior na variação destes índices.

\section{DISCUSSÃO}

Uma vez superada a alta mortalidade infantil como vem acontecendo nos países em desenvolvimento, a atenção de muitos pesquisadores voltou-se para as consequências do BPN sobre o desenvolvimento da criança. O BPN em RN a termo vem sendo descrito como importante fator de risco para o adequado desenvolvimento durante a infância, bem como, para uma elevada morbi-mortalidade infantil, devendo ser considerado relevante problema de saúde pública. Isto corre especialmente em países em desenvolvimento, onde a população é consideravelmente mais pobre e susceptível a fatores de risco biológico e ambiental $2,6,8,17-21$.

As crianças da coorte foram estudadas sob vários aspectos, entre eles o estudo do aleitamento materno, morbidade e desenvolvimento cognitivo ${ }^{18}$, além do acompanhamento do desenvolvimento mental e motor e os efeitos que o meio-ambiente exerce sobre os mesmos ${ }^{8}$. Em ambos os estudos demonstrou-se haver diferença estatisticamente significante no desenvolvimento destas crianças aos seis e doze meses, com piores resultados para o grupo de BPN em relação ao de PAN.

Por diversos motivos, não tem sido fácil comparar os dados da literatura quanto às complicações decorrentes do BPN. Neste contexto, Aylward et al. ${ }^{7}$, realizando uma meta-análise dos trabalhos publicados sobre o desenvolvimento neuropsicomotor em BPN na América do Norte, Europa, Austrália e Nova Zelândia, na década de 80 , sintetizaram os problemas metodológicos destes estudos em três principais áreas: critérios diferentes de seleção e amostragem, falha em controlar os possíveis fatores de confundimento e a não padronização dos resultados.

A maioria dos trabalhos sobre o tema analisada por Allen ${ }^{6}$ em sua meta-análise, mostrou, em crianças de BPN a termo, aumento da ocorrência de qua- 
dro clínico denominado na época de disfunção cerebral mínima, que incluía distúrbios neurológicos menores, déficit de atenção, atraso na linguagem e escolaridade insatisfatória, mesmo com nível cognitivo normal.

A avaliação do desenvolvimento da nossa amostra aos 24 meses confirmou os resultados encontrados já no primeiro ano de vida, mostrando, inclusive, ter havido ampliação da diferença destes índices com o progredir da idade, persistindo o desenvolvimento do grupo de BPN em desvantagem. O índice de desenvolvimento mental mostrou uma diferença de quatro pontos aos seis meses, sete pontos aos 12 meses e 9,1 pontos aos 24 meses, em detrimento do grupo de BPN. Tendência similar foi observada com o índice motor, com diferença de 7,3 pontos aos seis meses, 9,9 pontos aos 12 meses, chegando a 10,2 aos 24 meses $^{8}$.

O desenvolvimento mental e motor das crianças aos 24 meses permaneceu estatisticamente mais baixo no grupo de BPN, mesmo depois de ajustado pelo nível sócio-econômico. O BPN explicou 3\% da variação do índice mental $(p=0,01)$ e $5 \%$ da variação do índice motor $(p=0,005)$. Apesar do reduzido percentual de variação, este resultado demonstra a importância do peso ao nascer no desenvolvimento, corroborando a maioria das publicações sobre o tema ${ }^{7,8,11,12,19}$.

Como citado anteriormente, foi observada diferença significante de alguns indicadores sócio-econômicos e demográficos entre os dois grupos de crianças. Houve maior frequência de mães adolescentes e de baixa escolaridade no grupo de BPN. Segundo Blasco ${ }^{22}$, os pais são especialmente interessados pela motricidade de seus filhos no primeiro ano de vida. No nosso estudo, como a escolaridade materna esteve inicialmente associada ao desenvolvimento motor, pode-se especular o fato de que a mãe com nível de escolaridade melhor poderia ter maior conhecimento sobre o desenvolvimento infantil e estimularia mais seu bebê quanto à motricidade. No entanto, esta associação perdeu a sua significância na análise multivariada após a entrada de outras variáveis sócio-econômicas e de estimulação ambiental.

Embora não se tenha demonstrado diferença estatisticamente significante em relação à renda per capita entre os dois grupos, a posse de geladeira como variável que espelha o nível sócio-econômico da família, foi significantemente menos frequente no grupo de BPN. A posse de geladeira no domicílio expressando o nível sócio-econômico junto com a estimulação ambiental, foram responsáveis por $23 \%$ e $21 \%$ da variação do desenvolvimento mental e motor, respectivamente. Como esperado, já que os dados da literatura indicam aumento da importância das condições sócio-econômicas e demográficas com o passar da idade, estes fatores superaram a contribuição do BPN como fator determinante do desenvolvimento mental e motor aos 24 meses.

Aos 6 e 12 meses, constatou-se, através da avaliação do meio ambiente feita pelo Caldwell Home Inventory - $\mathrm{HOME}^{23}$, que o grupo de crianças de BPN recebeu uma estimulação ambiental mais pobre que o grupo de PAN e as variáveis analisadas estavam fortemente associadas ao desenvolvimento mental, mas não ao motor ${ }^{8}$.

A análise bivariada da estimulação ambiental referida pelas mães no segundo ano de vida mostrou que só o estímulo da habilidade motora fina, representada pelo incentivo à criança a pintar e/ou rabiscar, foi significantemente menos frequente no grupo de BPN. A pequena proporção de crianças que manipularam brinquedos nos dois grupos reflete $o$ baixo poder aquisitivo desta população, como também a falta de conhecimento das mães sobre o valor desta atividade para o desenvolvimento infantil.

Os múltiplos fatores determinantes do desenvolvimento infantil devem ser levados em consideração em programas de saúde. Ao contrário dos países afluentes, nos países pobres, o crescimento intrauterino adequado parece ter maior poder determinante sobre o desenvolvimento da criança, já que o meio ambiente não exerce função preventiva ou protetora, como nas sociedades desenvolvidas.

Estudos mais recentes, incluindo o presente trabalho, reforçam a hipótese de que tanto os riscos biológicos, entre eles o BPN, como as condições sócioeconômicas e ambientais influenciam o desenvolvimento pós-natal ${ }^{8,11,18,19,21}$. Apesar da grande heterogeneidade da população de BPN e da dificuldade em comparar os trabalhos, pode-se concluir que o RCIU aumenta o risco do déficit de desenvolvimento mental e motor, em especial na população pobre dos países em desenvolvimento. Embora possa parecer que essas alterações tenham pequena importância clínica, os autores sugerem que essas alterações sutis do desenvolvimento sejam levadas em consideração, uma vez que podem gerar importante diminuição da qualidade de vida desta população.

\section{REFERÊNCIAS}

1. World Health Organization. The incidence of low birth weight: an update. Weekly Epidem Rec 1984;59:205-211.

2. Kramer MS. Determinants of low birth weight: methodological assessment and meta-analysis. Bull WHO 1987;65:663-737. 
3. Lira PIC, Ashworth A, Morris SS. Low birth weight and morbidity from diarrhea and respiratory infection in northeast Brazil. J Pediatr 1996;128:497-504.

4. Monteiro CA. Estimativa dos coeficientes específicos de mortalidade infantil segundo o peso ao nascer no município de São Paulo (Brasil). Rev Saúde Pública 1981;15:603-610.

5. Barros FC, Huttly SR, Victora CG, Kirkwood BR, Vaughan JP. Comparison of causes and consequences of prematurity and intrauterine growth retardation: a longitudinal study in southern Brazil. Pediatrics 1992;90:238-244.

6. Allen MC. Developmental outcome and follow-up of the small for gestational age infants. Semin Perinatol 1984;8:123-156.

7. Aylward GP, Pfeiffer SI, Wright A, Verhulst SJ. Outcome studies of low birth weight infants published in the last decade: a metaanalysis. J Pediatr 1989;115:515-520.

8. Grantham-McGregor SM, Lira PIC, Ashworth A, Morris SS, Assunção MA. The development of low birthweight term infants and the effects of the environment in north-east Brazil. J Pediatr 1998;132:661-666.

9. Pollitt E. Developmental sequel from early nutrition deficiences: conclusive and probability judgements. Am Soc Nutr Sci 2000;Suppl.350S-353S.

10. The Scottish Low Birthweight Study Group. The Scottish low birth weight: I, survival, growth, neuromotor and sensory impairment. Arch Dis Child 1992;67:675-681.

11. Pollitt E, Golub M, Gorman K, et al. A reconceptualization of the effects of undernutrition on children's biological, psychosocial, and behavioral development. Social Policy Report 1996;10:1-21.

12. Grantham-McGregor SM, Walker SP, Chang SM, Powell CA. Effects of early supplementation with and without stimulation on later development in stunted Jamaicam children. Am J Clin Nutr 1997;66:247-253.

13. World Health Organization Working Group on Infant Growth. An evaluation of infant growth: the use and interpretation of anthropometry in infants. Bull WHO 1995;73:165-174.

14. Capurro H, Konichezki D, Fonseca D,Caldeyro-Barcia R. A simplified method for diagnosis of gestational age in the newborn infant. J Pediatr 1978;93:120-132.

15. Rhodes L, Bayley N, Yow BC. Bayley Scale of Infant Development versão 1984. Berkley: The Psychological Corporation, 1969.

16. World Health Organization. Guidelines for Assessing the Nutritional Impact of Supplementary Feeding Programmes for Vulnerable Groups. Geneva, 1983.

17. Crouse DT, Cassady G. The small for gestational age infant. In Avery GB, Fletcher MA, McDonald M (eds). Neonatology 4.Ed. Philadelphia: Lippincott, 1994:369-398.

18. Morris SS, Grantham-McGregor SM, Lira PIC, Assunção MA, Ashworth A. Effect of breastfeeding and morbidity on development of low birth weight term babies in Brazil. Acta Pediatr 1999;88:1101-1106.

19. Duncan GJ, Brooks-Gunn J, Klebanov PK. Economic deprivation and early childhood development. Child Dev 1994;65:296-318.

20. Garrett $\mathrm{P}, \mathrm{Ng}^{\prime}$ andu N, Ferron J. Poverty experiences of young children and the quality of their home environments. Child Dev 1994;65:331-345.

21. Brown JL, Pollitt E. Malnutrition, poverty and intellectual development. Sci Am 1996;144:38-43.

22. Blasco PA. Pitfalls in developmental diagnosis. Pediatr Clin N Am 23. Caldwell BM. Descriptive evaluations of child development and of developmental settings. Pediatrics 1967;40:46-50.

23. Caldwell BM. Descriptive evaluations of child development and of developmental settings. Pediatrics 1967;40:46-50. 